\title{
Astragaloside IV alleviates placental oxidative stress and inflammation in GDM mice
}

\author{
Ling Zhou, Ruixue Zhang, Shuangyan Yang, Yaguang Zhang and Dandan Shi \\ Cangzhou Central Hospital, Cangzhou, Hebei, China
}

Correspondence should be addressed to R Zhang: zhangruixue120@sina.com

\begin{abstract}
Background: Our previous study revealed that astragaloside IV (AS-IV) effectively improved gestational diabetes mellitus (GDM) by reducing hepatic gluconeogenesis. Due to the importance of placental oxidative stress, we further explored the protective role of AS-IV on placental oxidative stress in GDM.

Methods: First, non-pregnant mice were orally administrated with AS-IV to evaluate its safety and effect. Then GDM mice were orally administered with AS-IV for 20 days and its effect on the symptoms of GDM, placental oxidative stress, secretions of inflammatory cytokines, as well as toll-like receptor 4 (TLR4)/NF-kB signaling pathway, were evaluated. Results: AS-IV had no adverse effect on non-pregnant mice. On the other hand, AS-IV significantly attenuated the GDM-induced hyperglycemia, glucose intolerance, insulin resistance, placental oxidative stress, productions of inflammatory cytokines and the activation of TLR4/NF-kB pathway.

Conclusion: AS-IV effectively protected against GDM by alleviating placental oxidative stress and inflammation, in which TLR4/NF-кB might be involved.
\end{abstract} Key Words

$\begin{aligned} & \text { Key Words } \\ & \text { - } \text { AS-IV } \\ & \text { - } \text { placenta } \\ & \text { - oxidative stress } \\ & \text { - inflammation } \\ & \text { - } \text { TLR4NF-kB pathway } \\ & \text { - GDM }\end{aligned}$

\section{Introduction}

Gestational diabetes mellitus (GDM) is a condition of glucose intolerance usually occurring during the late stage of pregnancy due to decreased maternal insulin sensitivity and increased glucose production, and often recovers after delivery. Although it is not a chronic disease, without proper treatment, GDM would cause severe damage to the pregnant women and their fetus. GDM may cause polyhydramnios, gestational hypertension during pregnancy, and type II diabetes even after delivery. In terms of fetal outcome of the GDM patients, GDM increases the risks of fetal macrosomia, fetal growth restriction, intrauterine hypoxia or even stillbirth. Moreover, newborns from GDM patients are also prone to metabolic abnormalities.

Astragaloside IV (AS-IV) is one of the most effective components of stragalus membranaceus, and AS-IV is reported to have a wide range of pharmacological properties, including anti-inflammation, anti-oxidant and anti-hypertension, as well as improving immunity $(1,2,3)$, therefore AS-IV has been widely used to treat hypertension $(4,5)$, cardiovascular diseases $(6,7)$, breast cancer (8) and cervical cancer (9). Moreover, numerous publications have demonstrated that AS-IV could protect against diabetes and its complications, such as diabetic nephropathy, myocardial damage, diabetic wound healing and diabetic retinopathy $(6,10,11,12)$. Our group has investigated the effect of AS-IV in GDM-related diseases and discovered that AS-IV could effectively ameliorate GDM in genetic mouse model of GDM by inhibiting NLRP3 inflammasome in the pancreas (13). We also found that AS-IV remarkably reduced hepatic gluconeogenesis, hepatic inflammation and oxidative stress to alleviate GDM in mice (14). Although diabetes and GDM share similar pathophysiology, due to the complexity of pregnancy and the importance of oxidative stress and inflammation in the placenta, as well as the activation
This work is licensed under a Creative Commons Attribution-NonCommercial 4.0 International License. ded from Bioscientifica.com at 04/26/2023 01:01:57PM 
of toll-like receptor-4 (TLR4) and its downstream NF-кB signaling pathway, in this study, we aimed to explore the role of AS-IV on placental oxidative stress and the potential involvement of the TLR4 signaling pathway in GDM mice.

\section{Materials and methods}

\section{GDM mouse model}

C57BL/KsJ-Lep ${ }^{+/+}$(wild-type, wt) and C57BL/KsJ-Lep ${ }^{\mathrm{db} /+}$ $(\mathrm{db} /+)$ mice were all purchased from Shanghai Yunhao Biotech Company (Shanghai, China). We measured the food intake of wt and $\mathrm{db} /+$ mice, and found all the mice were pair-fed with the same amount of food (data not shown). To first study the effect of AS-IV on the role of non-pregnant mice, female $\mathrm{db} /+$ mice were randomly divided into two groups: one group was orally administrated with $20 \mathrm{mg} / \mathrm{kg}$ AS-IV (CrackAging, purity $>98 \%$ ) in saline solution daily, and the other group was orally administrated with the same amount of saline. The day before, the oral gavage was designated as day 0 . Blood was collected from the tail vein on days 0,10 and 20 , respectively.

Next, to investigate the effect of AS-IV on pregnant mice, female mice were individually mated with male mice of the same genetic background and mating was confirmed by the presence of a copulatory plug on the next morning, which was designated as gestation day (GD) 0. Pregnant mice were randomly divided into three groups of equal average body weight, with 16 mice in each group, and treated as previously described. Blood was collected and body weight was measured on GD 0 , 10 and 20, respectively. On GD 20, mice were sacrificed, and blood and placenta were collected. The study was approved by the ethics committee of Cangzhou Central Hospital.

\section{Glucose and insulin tolerance test}

On day 15 and GD 15, mice were subjected to glucose and insulin tolerance tests, respectively, as previously described (2). Briefly, after fasting for $6 \mathrm{~h}$, mice were intraperitoneally injected with glucose at a dose of $2 \mathrm{~g} / \mathrm{kg}$ body weight. Blood glucose levels were measured at the indicated time points after glucose injection using a glucometer (Roche Diagnostics). For the insulin tolerance test, mice were intraperitoneally injected with insulin at a dose of $1 \mathrm{mU} / \mathrm{kg}$ body weight after $1 \mathrm{~h}$ fasting; the following experiment was performed the same as the glucose tolerance test.

\section{Assessment of oxidative stress markers}

Reduced glutathione (GSH) was measured as described before (15), and GSH activity was shown as nM/mg of placental weight. The activities of catalase and glutathione peroxidase (GPx) were analyzed using commercial kits (Jiancheng Biological, Nanjing, China). Superoxide dismutase (SOD) activity was detected using a xanthine oxidase technique kit (ShanghaiSolarbio Bioscience and Technology Co., Shanghai, China).

\section{ELISA}

Interleukin-6 (IL-6), IL-1 $\beta$, tumor necrosis factor- $\alpha$ (TNF- $\alpha$ ) and serum insulin levels were measured using ELISA kits according to the manufacturer's instructions, respectively (AlPCO, France).

\section{Western blot}

Protein was extracted from lysed placenta in radioimmunoprecipitation lysis buffer on GD 20. After centrifuged at $12,000 \boldsymbol{g}$ at $4^{\circ} \mathrm{C}$ for $10 \mathrm{~min}$, protein was loaded into sodium dodecyl sulfate-polyacrylamide gel electrophoresis gel and transferred to a polyvinylidene fluoride membrane. After blocking in 5\% milk, the membrane was incubated with the primary antibody, and then with appropriate peroxidase-conjugated goat IgG secondary antibodies. All the antibodies were purchased from Santa Cruz. Blots were visualized by chemiluminescence (Thermo Fisher Scientific).

\section{qRT-PCR}

Total mRNA was extracted from lysed placenta of mice, and transcribed into cDNA by high-capacity cDNA reverse transcription kit (Thermo Fisher Scientific), then qRT-PCR was performed to detect PCR products by SYBR green (Molecular Probes) as previously described (16). The sequences of primers were as below:
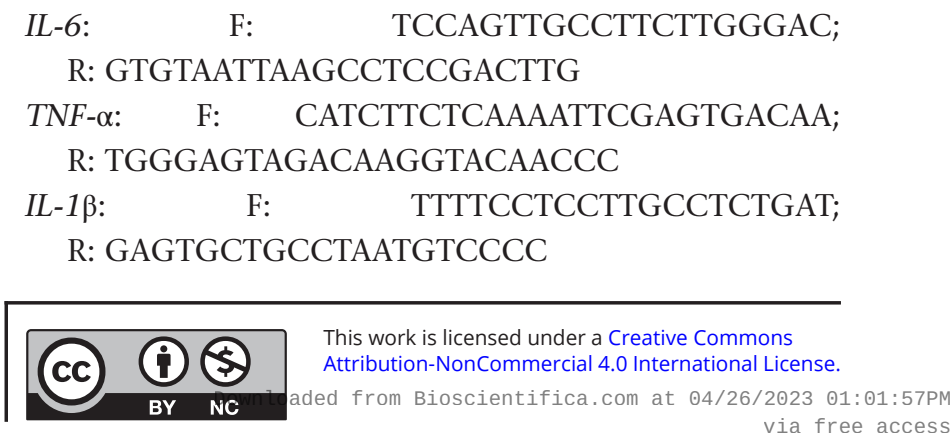


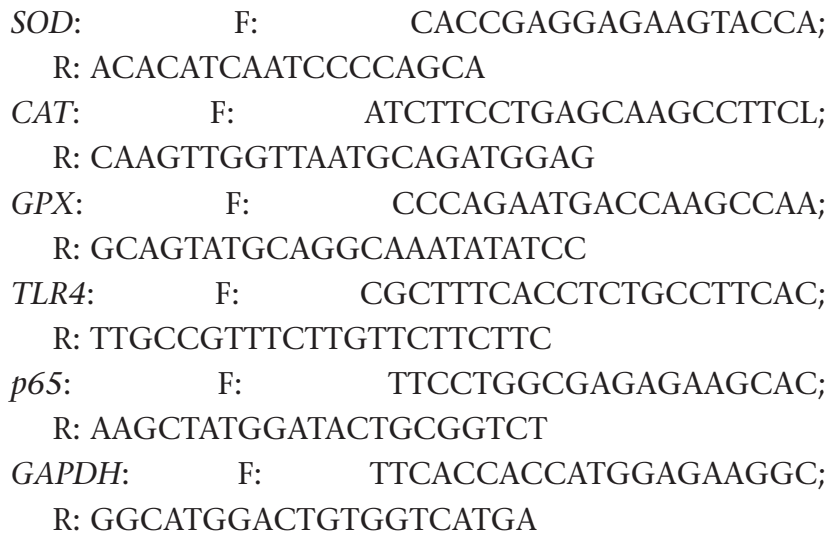

\section{Statistical analysis}

Data were analyzed by statistical product and service solutions (SPSS 16.0, SPSS Inc.) and expressed as mean \pm s.E.M. One-way or two-way analysis of variance followed by Bonferroni as post hoc test was used to analyze the difference.

\section{Results}

AS-IV had no effect on glucose levels, insulin levels, and glucose and insulin tolerance in non-pregnant mice

We treated non-pregnant mice with $20 \mathrm{mg} / \mathrm{kg}$ AS-IV daily for 20 days to confirm its safety, using the same dose reported in the previous study (17). First, we found that there was no difference in glucose levels, insulin levels, glucose or insulin tolerance between non-pregnant wt and $\mathrm{db} /+$ mice (Fig. 1A, B, C and D), which was consistent with previous publication showing that non-pregnant C57BL/KsJ-Lep ${ }^{\mathrm{db} /+}$ mice usually did not exhibit diabetic symptoms, such as hyperglycemia and insulin resistance (17). Nevertheless, AS-IV also had no effect on glucose levels, insulin levels, glucose or insulin tolerance in nonpregnant $\mathrm{db} /+$ mice. Considering that there were no death or obviously abnormal behavior in the mice, and the safety results tested in the previous publications, we concluded that this dose of AS-IV was safe in mice as previously described (17).

\section{AS-IV improved diabetic symptoms in pregnant $\mathrm{db} /+$ mice}

Next, we evaluated the role of AS-IV in pregnant $\mathrm{db} /+$ mice. Compared to wt mice, pregnant $\mathrm{db} /+$ mice exhibited
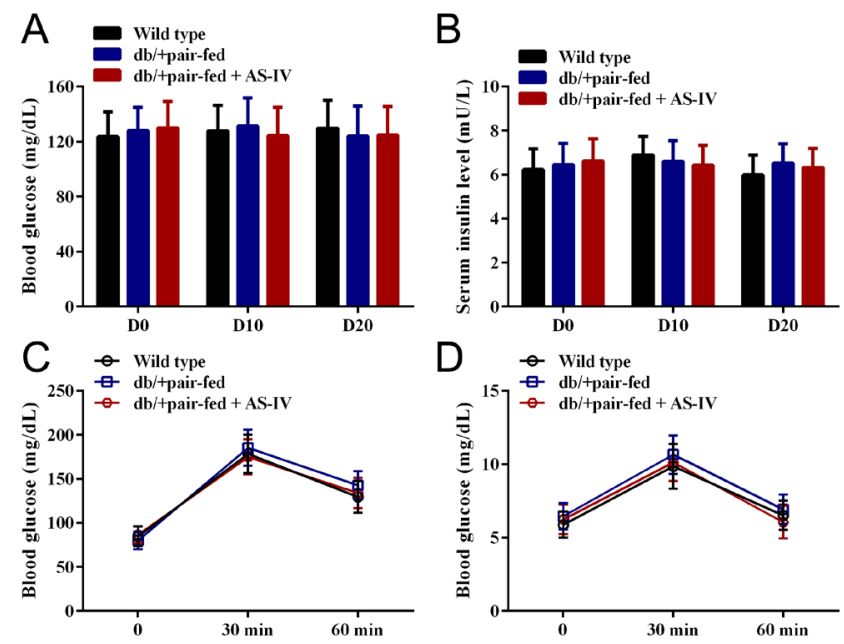

Nonpregnant mice

\section{Figure 1}

Astragaloside IV had no effect on glucose and insulin levels, glucose and insulin tolerance in nonpregnant mice. (A and B) The day before astragaloside IV fed was designated 0 days. The blood glucose and insulin levels were measured at 0,10 and 20 days. (C and D) Intraperitoneal glucose and insulin tolerance test were conducted on day 15 . The mice fasted for $6 \mathrm{~h}$ and intraperitoneal injection with glucose at $2 \mathrm{~g} / \mathrm{kg}$ or insulin at $0.75 \mathrm{U} / \mathrm{kg}$ body weight. The blood was collected from the tail vein after glucose or insulin injection for 0,30 , and $60 \mathrm{~min}$, and the glucose and insulin levels were determined. Data were presented as means \pm S.E.M. $n=8$ for each group.

the typical symptoms of GDM, including hyperglycemia (Fig. 2A), decreased serum insulin levels (Fig. 2B), glucose intolerance (Fig. 2C) and insulin resistance (Fig. 2D), all of which were significantly improved by the AS-IV treatment. Therefore, AS-IV could effectively attenuate the symptoms of GDM in pregnant $\mathrm{db} /+$ mice.

\section{AS-IV attenuated placental oxidative stress in the late stage of pregnancy in GDM mice}

Placental oxidative stress plays an important role in the development of GDM, therefore we evaluated the effect of AS-IV on placental oxidative stress in the late stage of pregnancy in GDM mice, by measuring oxidative stress markers, including malondialdehyde (MDA), which is the degradation product of reactive oxygen species (ROS), and ani-oxidant enzymes namely SOD, catalase (CAT), GPx and GSH. Compared to wt mice, GDM mice displayed an increased level of MDA (Fig. 3D) and decreased levels of anti-oxidative enzymes both in the serum and placenta on GD 20, including SOD (Fig. 3A and E), CAT (Fig. 3B), GPx (Fig. 3C and F) and GSH (Fig. 3G), indicating that placental oxidative stress was induced in GDM mice. Nevertheless, AS-IV treatment significantly attenuated the GDM-induced placental oxidative stress in the late stage of pregnancy.

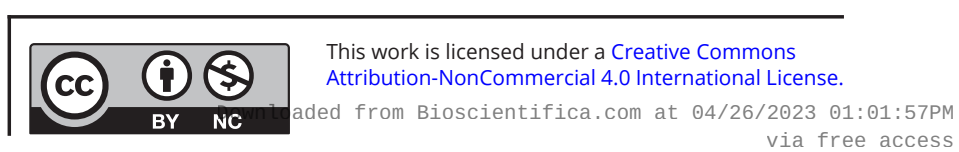



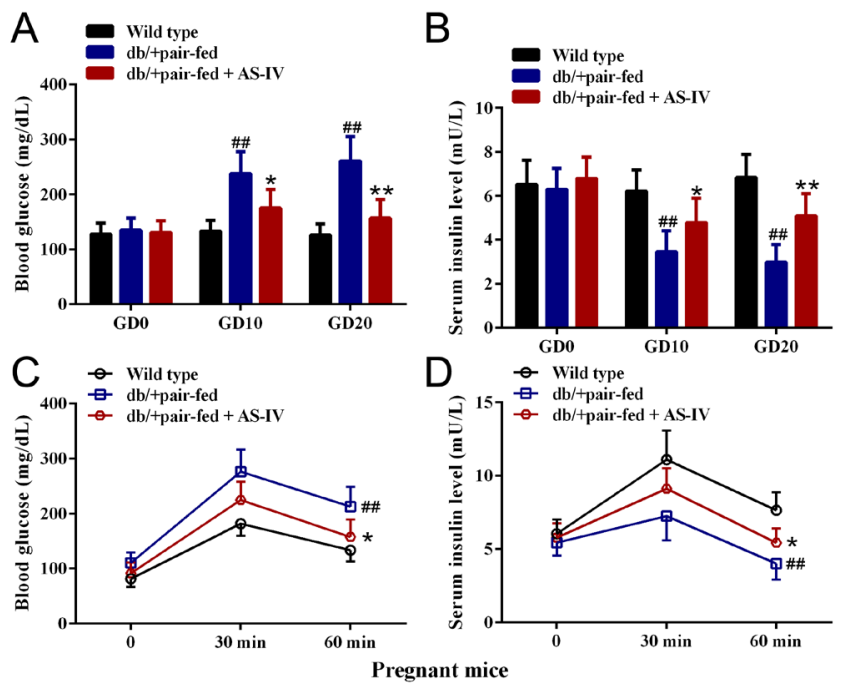

Figure 2

Astragaloside IV improved diabetic symptoms in pregnant $\mathrm{db} /+$ mice. ( $A$ and $B$ ) The day that female mice were successfully mated was designated gestation day (GD) 0 . The blood glucose and insulin levels were measured at 0,10 and 20 days. (C and D) Intraperitoneal glucose and insulin tolerance test were conducted on GD 15. The mice fasted for $6 \mathrm{~h}$ and intraperitoneal injection with glucose at $2 \mathrm{~g} / \mathrm{kg}$ or insulin at 0.75 $\mathrm{U} / \mathrm{kg}$ body weight. The blood was collected from the tail vein after glucose or insulin injection for 0,30, and 60 minutes, and the glucose and insulin levels were determined. Data were presented as means \pm S.E.M. $n=16$ for each group. ${ }^{\# \# P<0.01 \text { compared to wild type mice. }{ }^{*} P<0.05,{ }^{*} P P<0.01}$ compared to $\mathrm{db} /+$ pair-fed group.

\section{AS-IV reduced placental inflammation in the late stage of pregnancy in GDM mice}

Next, we explored the protective role of AS-IV in the inflammatory response in the late stage of pregnancy in GDM mice. Inflammation was enhanced in GDM mice on GD 20, as evidenced by the increased secretions of inflammatory cytokines both in the serum and placenta, including IL-6 (Fig. 4A and D), IL-1 $\beta$ (Fig. 4B and E) and TNF- $\alpha$ (Fig. 4C and F), all of which were significantly reduced by the treatment of AS-IV. Therefore, AS-IV could effectively reduce placental inflammation caused by GDM in the late stage of pregnancy.

\section{AS-IV inhibited placental TLR4/NF-KB pathway in the late stage of pregnancy in GDM mice}

Accumulating evidence revealed that AS-IV inhibited the inflammatory response through the TLR4/NF-kB signaling pathway $(2,18,19)$, which prompted us to speculate that AS-IV could also improve placental inflammation by inhibiting the TLR4/NF-кB pathway. In line with this, we observed that the TLR4/NF-кB pathway was activated in the GDM mice as evidenced by the increased protein and mRNA levels of TLR4 and p-p65 in the placenta of GDM mice (Fig. 5A, B, C, D, E and F), which were all inhibited by 20 days of AS-IV treatment. Taken together, AS-IV might suppress placental inflammation through the
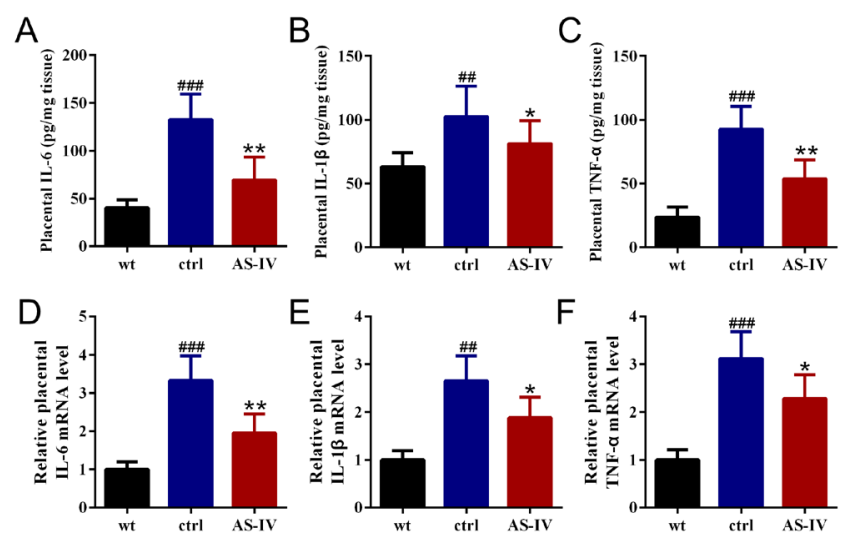

Figure 4

Astragaloside IV reduced placental inflammation in the late stage of pregnancy in GDM mice. ELISA was used to analyze the levels of IL-6 (A), $\mathrm{IL}-1 \beta(\mathrm{B})$ and TNF- $\alpha(C)$ in placenta on GD 20. qRT-PCR was used to analyzed the mRNA levels of IL- 6 (D), IL-1 $\beta$ (E) and TNF- $\alpha(F)$ in placenta on GD 20. GAPDH was set as a loading control and the relative expressions were normalized to wild type. Data were presented as means \pm S.E.M. $n=6$ for each group. ${ }^{\#} P<0.01,{ }^{\# \#} P<0.001$ compared to wild type mice. ${ }^{\star} P<0.05,{ }^{*} P<0.01$ compared to $\mathrm{db} /+$ pair-fed group. wt: wild type, ctrl: gestational diabetes mellitus mice (db/+ pair-fed), AS-IV: gestational diabetes mellitus mice treated with AS-IV. https://ec.bioscientifica.com https://doi.org/10.1530/EC-20-0295 (c) 2020 The authors Published by Bioscientifica Ltd
This work is licensed under a Creative Commons Attribution-NonCommercial 4.0 International License. ded from Bioscientifica.com at 04/26/2023 01:01:57PM

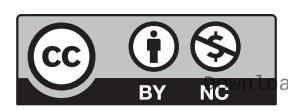

chioscientifica.com at 04/26/2023 01:01:57PM 
A
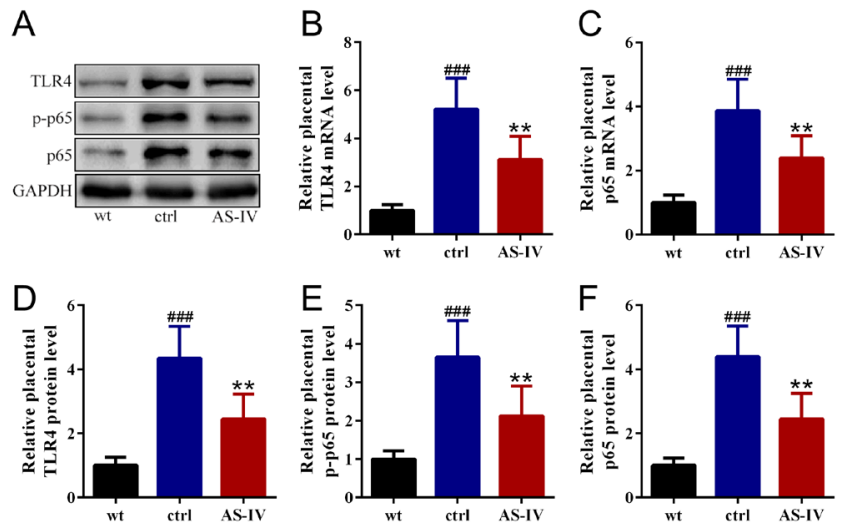

Figure 5

Astragaloside IV inhibited placental TLR4/NF-кB pathway in the late stage of pregnancy in GDM mice. Western blotting was used to assay the protein expressions of TLR4, p-p65 and p65 in placenta on GD 20 (A). GAPDH was set as a loading control and the relative expressions were normalized to wild type (D, E and F). qRT-PCR was used to analyzed the mRNA levels of TLR4 (B) and p65 (C) in placenta GD 20. GAPDH was set as a loading control and the relative expressions were normalized to wild type. Data were presented as means \pm S.E.M. $n=6$ for each group.

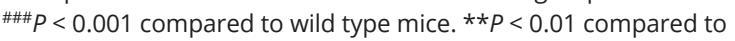
$\mathrm{db} /+$ pair-fed group. wt: wild type, ctrl: gestational diabetes mellitus mice (db/+ pair-fed), AS-IV: gestational diabetes mellitus mice treated with AS-IV.

TLR4/NF-кB signaling pathway in the late stage of pregnancy to ameliorate GDM symptoms.

\section{Discussion}

GDM is a common metabolic disorder, which occurs in more than $10 \%$ of pregnant women. Due the rapid improvement of life quality, the prevalence of GDM is also increasing with elevated incidence of obesity and the older maternal age worldwide. Although diabetes and GDM share similar pathophysiology, causes and symptoms, such as hyperglycemia, glucose intolerance and insulin resistance, the mechanism of GDM still needs to be further explored due to the complexity of pregnancy. Therefore, in recent years, increasing studies have focused on the investigation into novel therapeutic strategies for GDM (20).

AS-IV is the major active component of Astragalus membranaceus. Our group has studied AS-IV in the pathogenesis of many diseases for a long time, and discovered that AS-IV could effectively protect against GDM by inhibiting NLRP3 inflammasome in genetic GDM mice (13). Because of the multifunctional properties of AS-IV, we further investigated the protective effect of AS-IV against GDM and found that, AS-IV also attenuated hepatic gluconeogenesis to ameliorate GDM (14). Considering the importance of placental oxidative stress and inflammation during the pathogenesis of GDM, as well as the anti-oxidant and anti-inflammatory activities of AS-IV, we raised a hypothesis that AS-IV might ameliorate GDM by inhibiting placental oxidation and inflammation, therefore in the current study, we continued to explore the protective effect of AS-IV against GDM, and our data indicated that AS-IV effectively reduced placental oxidative stress and inflammation to alleviate GDM symptoms.

The placenta is an organ that only develops in the uterus during pregnancy. It connects the growing fetus via the umbilical cord to the uterine wall, allowing for nutrient uptake, thermoregulation, waste elimination and gas exchange through the blood supply of the mother, and protects against internal infection and produces essential hormones during pregnancy. Therefore, the placenta plays a crucial role during pregnancy, which is also involved in a variety of pregnancy-related diseases, including GDM. Numerous publications have reported that excessive ROS, produced as a result of increased placental oxidative stress, may be the underlying cause of insulin resistance, islet $\beta$-cell dysfunction and impaired glucose tolerance, therefore decreasing placental pro-oxidation could alleviate GDM $(21,22,23)$. However, unlike type 1 diabetes, patients with GDM could not develop a protective anti-oxidative mechanism to overcome the enhanced level of oxidative stress, therefore, additional supplement of anti-oxidant could be vital to attenuate the oxidative stress and to reduced complications of GDM both in the mother and the fetus (24). AS-IV, as a dual antioxidative and anti-inflammatory agent, could effectively inhibit both oxidative stress and inflammatory response in GDM mice, leading to attenuation of GDM symptoms.

In addition, TLR4, as well as its downstream NF- $\mathrm{B}$ signaling pathway, is involved in several physiological and pathological processes and regulates a wide range of diseases, particularly inflammation-related diseases such as GDM $(25,26,27,28)$. In patients with GDM, the expression of TLR 4 was increased in the peripheral blood mononuclear cells, with the activation of NF- $\mathrm{kB}$ signaling pathway as well as hyperglycemia and insulin resistance, and all of these factors participated in the development of GDM. Furthermore, inhibiting the activity of NF- $\mathrm{kB}$ could increase the expression of glucose transporter 4 (GLUT4) in the uterus of GDM rats, which indicated that GLUT4 expression reduced by the deactivation of NF- $\mathrm{B}$ might be relevant to the occurrence of GDM in the rat uterus due to insulin resistance. In addition, the activation of $\mathrm{NF}-\kappa \mathrm{B}$ also regulated cytokine secretion in the placenta of GDM rats. In summary, the NF- $\kappa B$ signaling pathway,

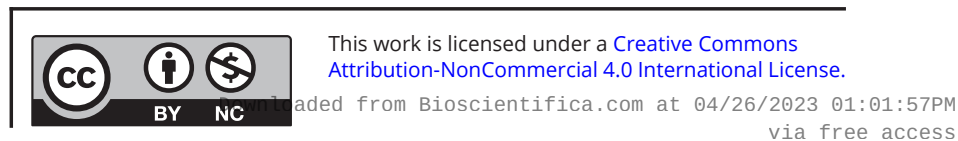


as well as its upstream TLR4, played an important role in the pathogenesis of GDM, therefore we investigated the role of TLR4/NF-кB signaling pathway in AS-IVtreated GDM mice, and our data demonstrated that the TLR4/NF-kB signaling pathway mediated the process of AS-IV-attenuated GDM symptoms.

In summary, following the previous studies on AS-IV in GDM mice, we further explored the placental oxidative stress, placental inflammation and the TLR4/NF- $\mathrm{KB}$ signaling pathway in the process of AS-IV-ameliorated GDM. Our data suggested that AS-IV could effectively reduce both the placental oxidative stress and placental inflammation to ameliorate GDM in genetic GDM mice, in which the TLR4/NF-kB signaling pathway might be involved. Therefore, our results suggested another detailed mechanism of AS-IV on GDM, which provided more evidence for utilizing AS-IV in clinical trials among patients with GDM. Our studies on the effect of AS-IV on GDM mice further suggested that AS-IV might serve as a novel therapeutic candidate against GDM.

\section{Conclusion}

AS-IV significantly reduced both the placental oxidative stress and placental inflammation in GDM mice, in which the TLR4/NF- $\mathrm{kB}$ signaling pathway might be involved. Therefore, AS-IV might serve as a novel therapeutic candidate against GDM.

\section{Declaration of interest}

The authors declare that there is no conflict of interest that could be perceived as prejudicing the impartiality of the research reported.

\section{Funding}

This work did not receive any specific grant from any funding agency in the public, commercial, or not-for-profit sector.

\section{References}

1 Zhuang Z, Wang ZH, Deng LH, Zheng Q, Zheng GQ \& Wang Y. Astragaloside IV exerts cardioprotection in animal models of viral myocarditis: a preclinical systematic review and meta-analysis. Frontiers in Pharmacology 201910 1388. (https://doi.org/10.3389/ fphar.2019.01388)

2 Chen JK, Guo MK, Bai XH, Chen LQ, Su SM, Li L \& Li JQ. Astragaloside IV ameliorates intermittent hypoxia-induced inflammatory dysfunction by suppressing MAPK/NF-kappaB signalling pathways in Beas-2B cells. Sleep and Breathing 202024 1237-1245. (https://doi.org/10.1007/s11325-019-01947-8)

3 Ding Q, Gao J, Zheng J, Wang A \& Jing S. Astragaloside IV attenuates inflammatory injury and promotes odontoblastic differentiation in lipopolysaccharide-stimulated MDPC-23 cells and rat pulpitis Journal of Oral Pathology and Medicine 201948 951-958. (https://doi. org/10.1111/jop.12926)

4 Zhang X, Chen J, Xu P \& Tian X. Protective effects of astragaloside IV against hypoxic pulmonary hypertension. MedChemComm 20189 1715-1721. (https://doi.org/10.1039/c8md00341f)

5 Jiang P, Ma D, Wang X, Wang Y, Bi Y, Yang J, Wang X \& Li X. Astragaloside IV prevents obesity-associated hypertension by improving pro-inflammatory reaction and leptin resistance. Molecules and Cells 201841 244-255. (https://doi.org/10.14348/ molcells.2018.2156)

6 Zhang Z, Wang J, Zhu Y, Zhang H \& Wang H. Astragaloside IV alleviates myocardial damage induced by type 2 diabetes via improving energy metabolism. Molecular Medicine Reports 201920 4612-4622. (https://doi.org/10.3892/mmr.2019.10716)

7 Yang P, Zhou YP, Chang XC, Wang F \& Li GW. Astragaloside regulates Nrf2/Bach1/HO-1 signaling pathway and inhibits H9c2 cardiomyocyte injury induced by hypoxia-reoxygenation. Zhongguo Zhong yao za zhi 201944 2331-2337. (https://doi.org/10.19540/j. cnki.cjcmm.20190312.001)

8 Zhang XQ, Yao C, Bian WH, Chen X, Xue JX, Zhu ZY, Ying Y, $\mathrm{Xu}$ YL \& Wang C. Effects of astragaloside IV on treatment of breast cancer cells execute possibly through regulation of Nrf2 via PI3K/ AKT/mTOR signaling pathway. Food Science and Nutrition 20197 3403-3413. (https://doi.org/10.1002/fsn3.1154)

9 Zhang L, Zhou J, Qin X, Huang H \& Nie C. Astragaloside IV inhibits the invasion and metastasis of SiHa cervical cancer cells via the TGFbeta1mediated PI3K and MAPK pathways. Oncology Reports 2019 41 2975-2986. (https://doi.org/10.3892/or.2019.7062)

10 Fan Y, Fan H, Zhu B, Zhou Y, Liu Q \& Li P. Astragaloside IV protects against diabetic nephropathy via activating eNOS in streptozotocin diabetes-induced rats. BMC Complementary and Alternative Medicine 201919 355. (https://doi.org/10.1186/s12906-019-2728-9)

11 Luo X, Huang P, Yuan B, Liu T, Lan F, Lu X, Dai L, Liu Y \& Yin H. Astragaloside IV enhances diabetic wound healing involving upregulation of alternatively activated macrophages. International Immunopharmacology 201635 22-28. (https://doi.org/10.1016/j. intimp.2016.03.020)

12 Ding Y, Yuan S, Liu X, Mao P, Zhao C, Huang Q, Zhang R, Fang Y, Song $\mathrm{Q}$, Yuan $\mathrm{D}$, et al. Protective effects of astragaloside IV on $\mathrm{db} / \mathrm{db}$ mice with diabetic retinopathy. PLoS ONE 20149 e112207. (https:// doi.org/10.1371/journal.pone.0112207)

13 Zhang R, Zhang X, Xing B, Zhao J, Zhang P, Shi D \& Yang F. Astragaloside IV attenuates gestational diabetes mellitus via targeting NLRP3 inflammasome in genetic mice. Reproductive Biology and Endocrinology 201917 77. (https://doi.org/10.1186/s12958-019-0522-7)

14 Zhang R, Xing B, Zhao J, Zhang X, Zhou L, Yang S, Wang Y \& Yang F. Astragaloside IV relieves gestational diabetes mellitus in genetic mice through reducing hepatic gluconeogenesis. Canadian Journal of Physiology and Pharmacology 202098 466-472. (https://doi. org/10.1139/cjpp-2019-0548)

15 Fu J, Fu J, Yuan J, Zhang N, Gao B, Fu G, Tu Y \& Zhang Y. Antidiabetic activities of Acanthopanax senticosus polysaccharide (ASP) in combination with metformin. International Journal of Biological Macromolecules 201250 619-623. (https://doi.org/10.1016/j. ijbiomac.2012.01.034)

16 Liu Z, Luo H, Zhang L, Huang Y, Liu B, Ma K, Feng J, Xie J, Zheng J, Hu J, et al. Hyperhomocysteinemia exaggerates adventitial inflammation and angiotensin II-induced abdominal aortic aneurysm in mice. Circulation Research 2012111 1261-1273. (https:// doi.org/10.1161/CIRCRESAHA.112.270520)

17 Song H, Xu Y, Yang X, Rong X, Wang Y \& Wei N. Tertiary butylhydroquinone alleviates gestational diabetes mellitus in C57BL/KsJ-Lep db/+ mice by suppression of oxidative stress. Journal of Cellular Biochemistry 2019120 15310-15319. (https://doi. org/10.1002/jcb.28798) https://ec.bioscientifica.com https://doi.org/10.1530/EC-20-0295 (c) 2020 The authors Published by Bioscientifica Ltd
This work is licensed under a Creative Commons Attribution-NonCommercial 4.0 International License. ded from Bioscientifica.com at 04/26/2023 01:01:57PM 
18 Wang N, Zhang X, Ma Z, Niu J, Ma S, Wenjie W \& Chen J. Combination of tanshinone IIA and astragaloside IV attenuate atherosclerotic plaque vulnerability in ApoE(-/-) mice by activating PI3K/AKT signaling and suppressing TRL4/NF-kappaB signaling. Biomedicine and Pharmacotherapy 2019123 109729. (https://doi. org/10.1016/j.biopha.2019.109729)

19 Zhang X, Li M \& Wang H. Astragaloside IV alleviates the myocardial damage induced by lipopolysaccharide via the toll-like receptor 4 (TLR4)/nuclear factor kappa B (NF-kappaB)/proliferator-activated receptor alpha (PPARalpha) signaling pathway. Medical Science Monitor 201925 7158-7168. (https://doi.org/10.12659/MSM.916030)

20 Yu B, Liu Z, Fu Y, Wang Y, Zhang L, Cai Z, Yu F, Wang X, Zhou J \& Kong W. CYLD deubiquitinates nicotinamide adenine dinucleotide phosphate oxidase 4 contributing to adventitial remodeling. Arteriosclerosis, Thrombosis, and Vascular Biology 201737 1698-1709. (https://doi.org/10.1161/ATVBAHA.117.309859)

21 Coughlan MT, Vervaart PP, Permezel M, Georgiou HM \& Rice GE. Altered placental oxidative stress status in gestational diabetes mellitus. Placenta 200425 78-84. (https://doi.org/10.1016/S01434004(03)00183-8)

22 Biri A, Onan A, Devrim E, Babacan F, Kavutcu M \& Durak I. Oxidant status in maternal and cord plasma and placental tissue in gestational diabetes. Placenta 200627 327-332. (https://doi. org/10.1016/j.placenta.2005.01.002)

23 Lappas M, Hiden U, Desoye G, Froehlich J, Hauguel-de Mouzon S $\&$ Jawerbaum A. The role of oxidative stress in the pathophysiology of gestational diabetes mellitus. Antioxidants and Redox Signaling 201115 3061-3100. (https://doi.org/10.1089/ ars.2010.3765)

24 Chen X \& Scholl TO. Oxidative stress: changes in pregnancy and with gestational diabetes mellitus. Current Diabetes Reports 20055 282-288. (https://doi.org/10.1007/s11892-005-0024-1)

25 Feng H, Su R, Song Y, Wang C, Lin L, Ma J \& Yang H. Positive correlation between enhanced expression of TLR4/MyD88/ NF-kappaB with insulin resistance in placentae of gestational diabetes mellitus. PLOS ONE 201611 e0157185. (https://doi. org/10.1371/journal.pone.0157185)

26 Kuzmicki M, Telejko B, Wawrusiewicz-Kurylonek N, Lipinska D, Pliszka J, Wilk J, Zielinska A, Skibicka J, Szamatowicz J, Kretowski A, et al. The expression of genes involved in NF-kappaB activation in peripheral blood mononuclear cells of patients with gestational diabetes. European Journal of Endocrinology 2013168 419-427. (https://doi.org/10.1530/EJE-12-0654)

27 Wei B, Ta N, Li J, Zhang JF \& Chen BL. Relationship between the inhibition of NF-kappaB and insulin resistance in uterrus of rats with gestational diabetes mellitus. Xi Bao Yu Fen Zi Mian Yi Xue Za Zhi 201026 235-247, 241.

28 Coughlan MT, Permezel M, Georgiou HM \& Rice GE. Repression of oxidant-induced nuclear factor-kappaB activity mediates placental cytokine responses in gestational diabetes. Journal of Clinical Endocrinology and Metabolism 200489 3585-3594. (https://doi. org/10.1210/jc.2003-031953)

Received in final form 27 August 2020 Accepted 3 September 2020

Accepted Manuscript published online 4 September 2020
This work is licensed under a Creative Commons Attribution-NonCommercial 4.0 International License. ded from Bioscientifica.com at 04/26/2023 01:01:57PM 